Research in Astronomy and Astrophysics manuscript no.

(ETTEX: tikhonov' 0. tex; printed on November 11,$2020 ; 1: 44$ )

Key words: stars: massive, stars: variables: LBV, galaxies: individual: M 94, DDO 68, NGC 1672

\title{
Search for the brightest stars in galaxies outside the Local Group *
}

\author{
N.A. Tikhonov ${ }^{1}$, O.A. Galazutdinova ${ }^{1}$, O.N. Sholukhova ${ }^{1}$, A. Valcheva ${ }^{2}$, P.L. Nedialkov ${ }^{2}$, \\ O.A. Merkulova ${ }^{3}$ \\ ${ }^{1}$ Special Astrophysical Observatory, Nizhnij Arkhyz, Karachai-Cherkessian Republic, Russia 369167; \\ ntik@sao.ru \\ 2 Department of Astronomy, Sofia University, Sofia, Bulgaria, 1504 Sofia, 15 Tsar Osvoboditel Blvd. \\ 3 Astronomical Institute, St.Petersburg State University, Universitetskii pr. 28, St.Petersburg, 198504 \\ Russia Received 2019 November 15; accepted 20xx month day
}

\begin{abstract}
This paper shows a technique for searching for bright massive stars in galaxies beyond the Local Group. To search for massive stars, we used the results of stellar photometry of the Hubble Space Telescope images using the DAOPHOT and DOLPHOT packages. The results of such searches are shown on the example of the galaxies DDO 68, M94 and NGC 1672. In the galaxy DDO 68 the LBV star changes its brightness, and in M 94 massive stars can be identified by the excess in the $\mathrm{H} \alpha$ band. For the galaxy NGC 1672, we measured the distance for the first time by the TRGB method, which made it possible to determine the luminosities of the brightest stars, likely hypergiants, in the young star formation region. So far we have performed stellar photometry of HST images of 320 northern sky galaxies located at a distance below $12 \mathrm{Mpc}$. This allowed us to identify 53 galaxies with probable hypergiants. Further photometric and spectral observations of these galaxies are planned to search for massive stars.
\end{abstract}

\section{INTRODUCTION}

One of the main tasks of modern astrophysics is to determine the upper limit of the mass of a star. The interest lies in the fact that massive stars evolve quickly and the final stage of their evolution can be a supernova burst, formation of a black hole or a neutron star, or even a merger of such relativistic objects with the release of huge amount of energy. The evolutionary path of a star depends on its initial mass. Models of stellar evolution suggest that the initial masses of stars can reach up to 500 solar masses (Yusof et al. 2013; Spera \& Mapelli 2017) and more, but so far, only the stars of much smaller mass have been discovered (Crowther et al. 2010). The question of the fidelity of theoretical models can only be solved by searching for and studying massive stars in galaxies of various masses.

In our Galaxy, massive stars are observed in clusters located in the galactic plane, where the extinction of light can be very high (Eikenberry et al. 2004). To increase the breadth of the search and solve the

$*$ Based on observations with the NASA/ESA Hubble Space Telescope, obtained at the Space Telescope Science Institute, which is operated by AURA, Inc. under contract No. NAS5-26555. These observations are associated with proposal 
problem with the uncertainty of light extinction, one should go beyond the Galaxy and search for massive stars in other, not too distant galaxies (Humphreys et al. 2017; Sholukhova et al. 2018). Using the Hubble Space Telescope, numerous galaxies outside the Local Group are accessible for the study (up to distances of $15-20 \mathrm{Mpc})$.

\section{SEARCH FOR CANDIDATES FOR MASSIVE STARS}

Spectral observations make it possible to determine the type of star and evaluate its physical parameters, but such observations are not suitable for search tasks because of the large number of candidates for massive stars and high time expenditures to obtain the results. Therefore, to identify possible candidates for massive stars (hypergiants), photometric methods are used, as they are faster and more accessible on all telescopes.

It seems that finding massive stars, which are also the brightest stars in galaxies, is not difficult, but this opinion is incorrect. In many low-mass galaxies, there is simply no such stars. The lifetime of these stars is very small (several million years), and the frequency of their appearance in galaxies is small. According to the law of mass distribution of Salpeter, thousands of stars of smaller masses should be born per star with a mass of 100 Solar masses. Even in our giant Galaxy, with numerous of star formation regions, only a few stars with masses of 150—300 Solar masses are known: HD15558A (Garmany \& Massey 1981), Eta Carinae (Gull 2015).

The second factor that complicates the search is the superposition of stars of our Galaxy on the studied fields. An example is given by the metal-poor dwarf galaxy DDO68 (Figure 1), the distance to which is $12 \pm 0.3 \mathrm{Mpc}$ (Tikhonov et al. 2014). In this galaxy, a bright massive LBV (Luminous Blue Variable) star is known (Pustilnik et al. 2017), which is marked on the image of the galaxy (Figure 1). On the HertzsprungRussell diagram (CM-diagram) DDO 68 (Figure 2), this star is very bright (according to our measurements, $\left.M_{V}=-10.26\right)$. However, this CM-diagram contains another brighter blue star, also visible on the body of the galaxy (Figure 1). As shown by spectral observations with 6-m telescope BTA, this bright blue star has no emission lines, and its radial velocity is low compared to the DDO 68. This indicates that it is not a massive star DDO 68, but belongs to our Galaxy. Isochrones of Bertelli et al. (1994) overploted on the DDO 68 CM-diagram also show that the bright blue star does not belong to the stars of the DDO 68. Thus, the probability of a foreground star falling into the selection of candidates for massive stars is far from zero. A convenient feature for identifying foreground stars is to compare the positions of bright stars with respect to star formation regions. The probability that a massive star will appear in a star cluster is always higher than the probability of its isolated appearance. A comparison of the positions of two bright blue stars in the DDO 68 galaxy shows that the LBV star is indeed surrounded by a cluster of stars and gas clouds, while the foreground star is completely isolated. However, this feature is not absolutely mandatory, since bright massive stars are known outside the clusters.

The third factor that impedes the search is the insufficient spatial resolution of the telescopes. Even in our Galaxy, when using ground-based telescopes, it is not possible to separate close groups of massive stars into separate stars. Therefore, when the mass of the entire group of stars is attributed to a single star, errors in mass estimation can occur. The Hubble Space Telescope allows to separate close groups and evaluate the 


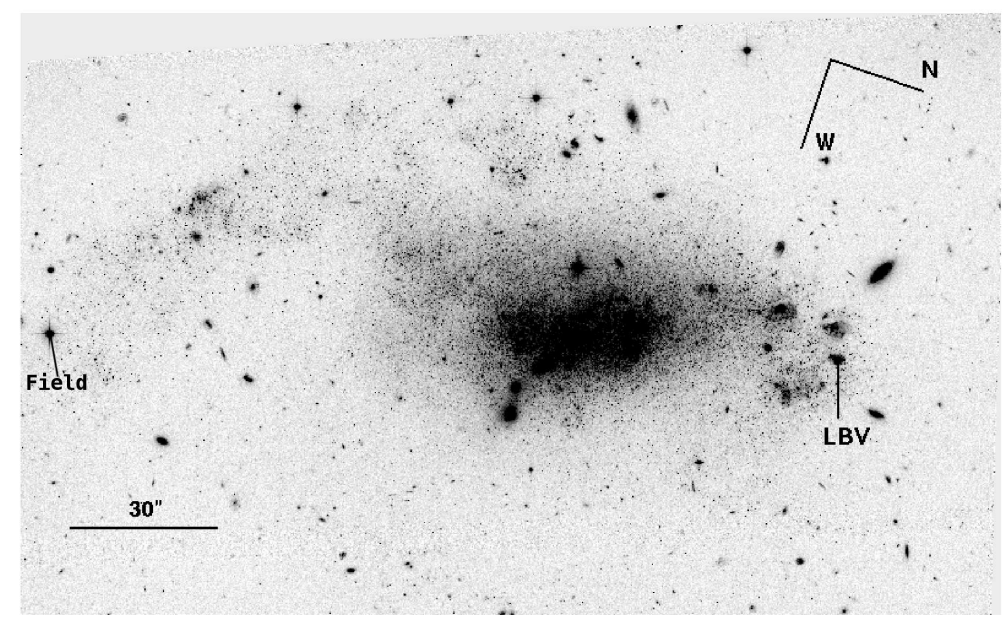

Fig. 1: Dwarf metal-poor galaxy DDO 68 in the F606W $(V)$ filter with the LBV star, marked by a circle and a bright blue field star belonging to our Galaxy.

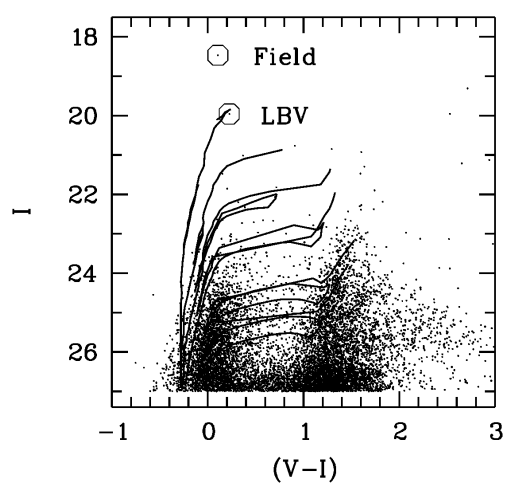

Fig. 2: CM-diagram of DDO68 stars and isochrones of age from 3 Myr to $100 \mathrm{Myr}$ and metalicity $Z=0.001$ from Bertelli et al. (1994). The position of the LBV star and the foreground star is marked.

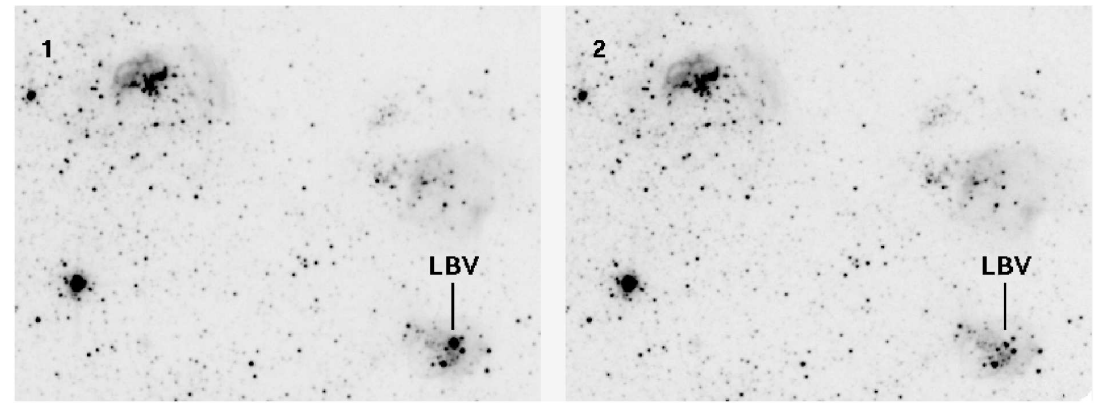

Fig. 3: Images of the galactic region DDO 68 in 2010 and 2017 in the F606W filter, in which the LBV star is marked by a line. A large change in the luminosity of the LBV star over 7 years can be seen.

The unstable state of massive stars often leads to variability of their brightness, which can be used for search of such stars. However, the constancy of the brightness of a star over several years does not mean its stability for a longer time interval. Only long series of accurate photometric measurements can give an answer about brightness variability. Figure 3 shows the images of LBV star in DDO 68 in 2010 and 2017. The pictures clearly show a strong drop in its brightness from $V=20^{\mathrm{m}} \cdot 19$ (2010) to $V=23{ }^{\mathrm{m}} \cdot 70$ (2017) 


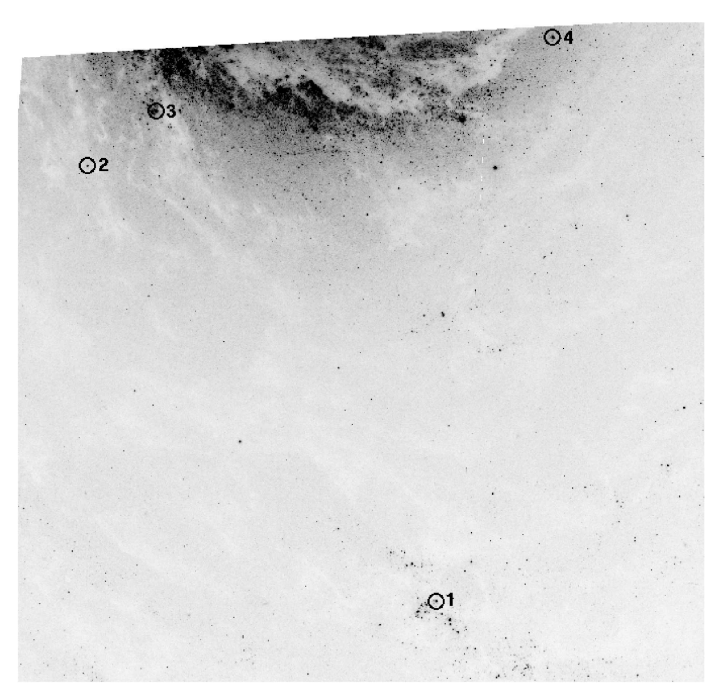

Fig. 4: Part of the galaxy M94 in HST image in the F555W filter. The positions of four bright blue stars with increased brightness in the $\mathrm{H} \alpha$ filter are marked.
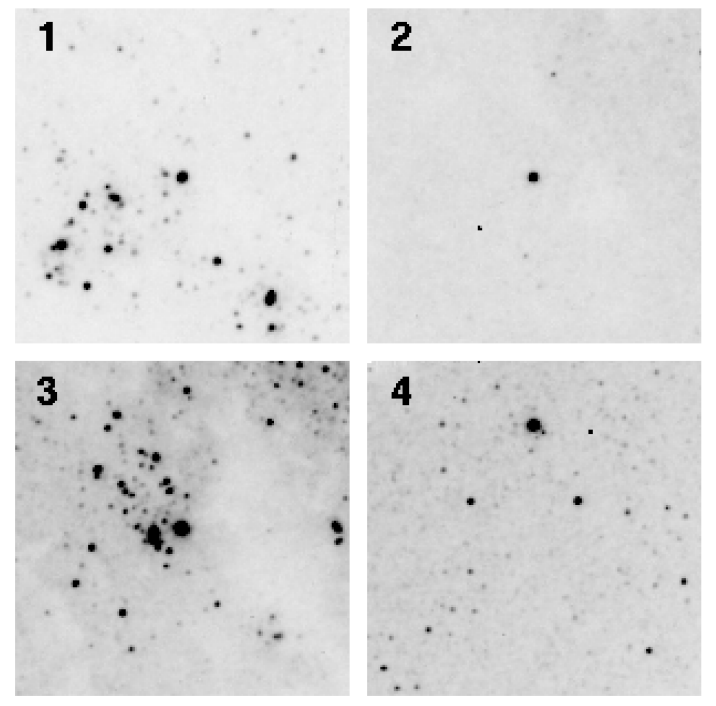

Fig. 5: Regions of stars marked in Fig. 4 with numbers. Star N4 is shown off-center.

Due to the instability of massive stars, gas loss occurs and various shells and clouds form. In the spectra of such stars, a strong $\mathrm{H} \alpha$ hydrogen emission line should be observed. The presence of the emission line greatly simplifies the search of candidates for massive stars. Images of the galaxy in $\mathrm{H} \alpha$ filter, together with images in other filters (F435W, F555W, F606W and F814W) allow to highlight bright blue stars with H $\alpha$ emission, which are likely to be the sought-after massive stars. But even after such a selection, extraneous objects fall into the list. Young compact star clusters almost do not differ in profile from single stars; they have a strong $\mathrm{H} \alpha$ emission and a small color index, identical to the color index of bright massive stars. High 


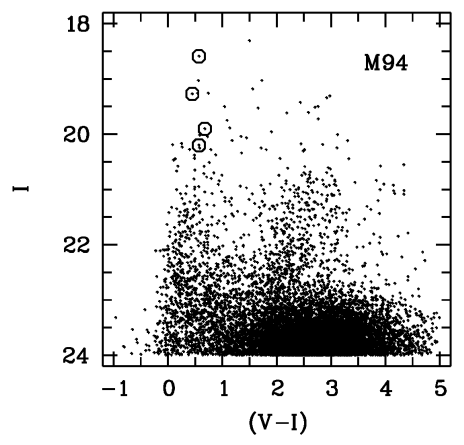

Fig. 6: CM diagram of the field of the galaxy M94. Among the bright blue objects are both massive stars and possible young compact clusters.

\section{SEARCH FOR MASSIVE STARS IN THE GALAXIES M94 AND NGC 1672}

Figure 4 shows an HST image of a part of the galaxy M94. The stellar photometry of this galaxy was performed with DOLPHOT 2.0 software package ${ }^{1}$. The DOLPHOT 2.0 package was used in accordance with Dolphin's recommendations (Dolphin 2016), while the photometry procedure consisted of bad pixel premasking, cosmic-ray particle hit removal, and further PSF photometry for the stars found in two filters. The photometry parameters used in our work can be found in the reference ${ }^{2}$. The obtained results of stellar photometry were selected according to the $C H I<1.3$ and $|S H A R P|<0.3$ parameters, which define the shape of the photometric profile of each measured star. This allowed us to remove all diffuse objects (star clusters, distant or compact galaxies) from the photometry tables, because the photometric profiles of these objects differed from those of stars.

After applying the DOLPHOT 2.0 package to the images of the WFC3/UVIS camera, we get the results of VegaMag photometry, which differ from Johnson-Cousins system. However, when DOLPHOT 2.0 photometry of the ACS camera images, we obtain results for the stars, both VegaMag and Johnson-Cousins systems. Therefore, it is not difficult to translate magnitudes from one system to another. In addition, for blue LBV stars, the differences between the systems are very small and do not even exceed the photometry error (i.e., less than $0{ }^{\mathrm{m}} 02$ ).

We tested a method for identifying probable massive stars based on photometry of galaxies in several filters. The archive HST telescope contains images of the M94 galaxy (ID10402) obtained in the F435W, F555W, F658N and F814W filters with exposures of 1200, 1000, 1400 and 900 seconds. In this galaxy, a visual search for massive stars has already been carried out and three probable LBV stars have been found (Solovyeva et al. 2019).

The Hertzsprung-Russell diagram obtained after photometry of stars the M94 is shown in Figure 6. Bright blue stars are highlighted on the diagram. An additional diagram using photometry in the $\mathrm{H} \alpha$ filter (Figure 7) makes it possible to distinguish 4 blue stars with the possible presence of $\mathrm{H} \alpha$ emission lines in the spectrum. The regions of these stsrs are shown on an enlarged scale in (Figure 5).

\footnotetext{
${ }^{1}$ http://americano.dolphinsim.com/dolphot/dolphot.pdf
} 


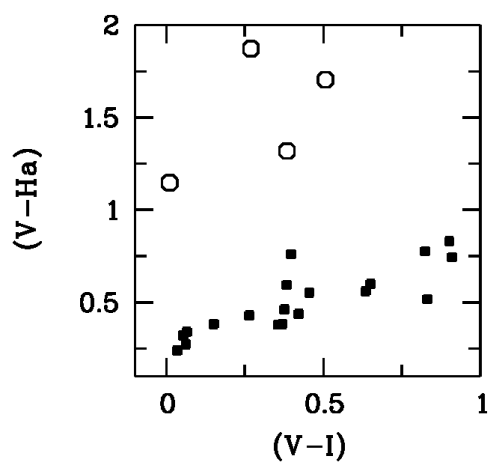

Fig. 7: CM diagram of bright blue stars seen in the diagram in Fig. 6. Black squares denote stars without emission in the $\mathrm{H} \alpha$ line, and circles - stars with emission. These stars are marked on the image of the galaxy (Fig. 4).

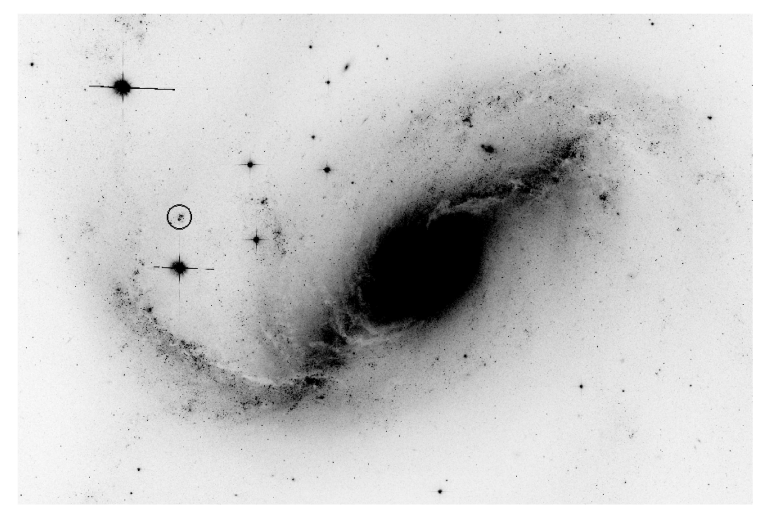

Fig. 8: Galaxy NGC 1672 in HST image in the F814W filter. The circle marks the position of the gas and dust nebula and star cluster containing stars up to $M_{V}=-10$.
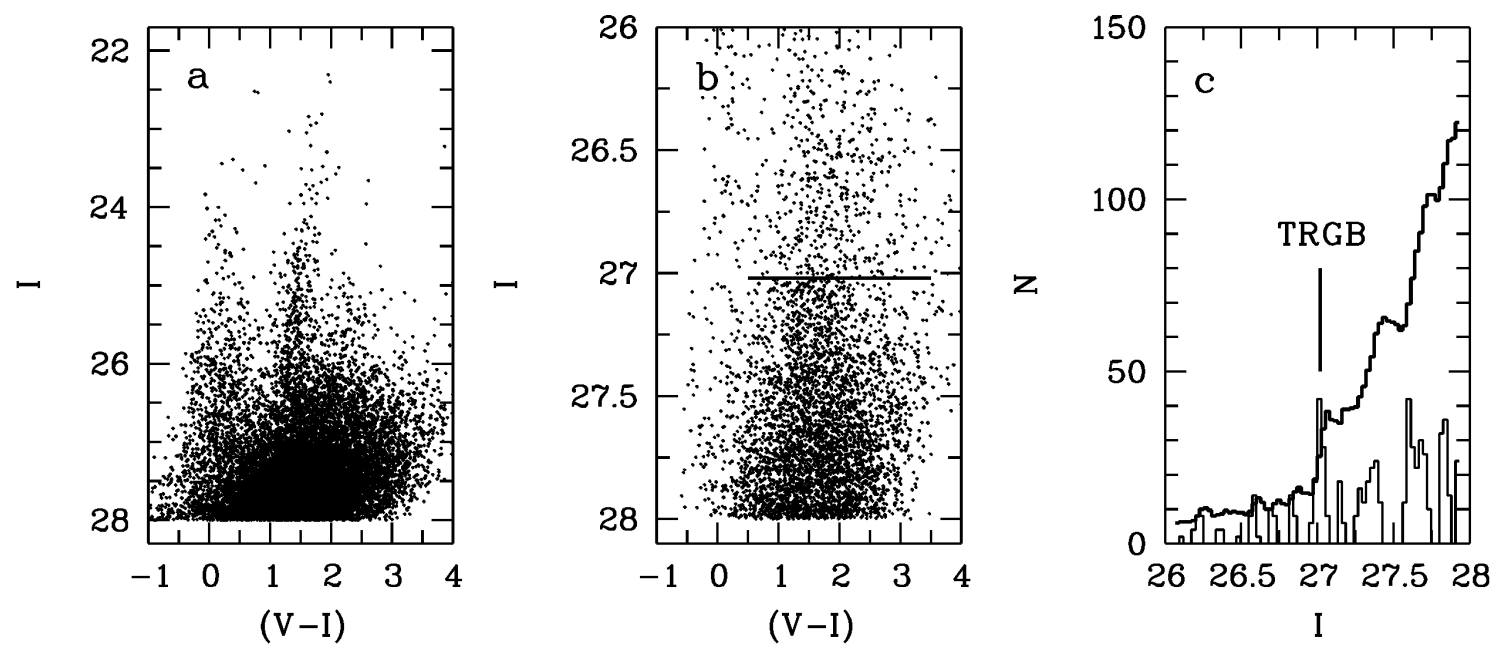

Fig. 9: CM diagram of stars in the periphery of NGC 1672 (a) and CM diagram of the same field (b) after the removal of stars from star-formation regions. The luminosity function of red giants and AGB stars (c) was obtained after selection stars for the color index $(V-I)$. The thin line shows the Sobel function, showing the increase in the number of stars (TRGB jump) at $I=27 \cdot 02$. 

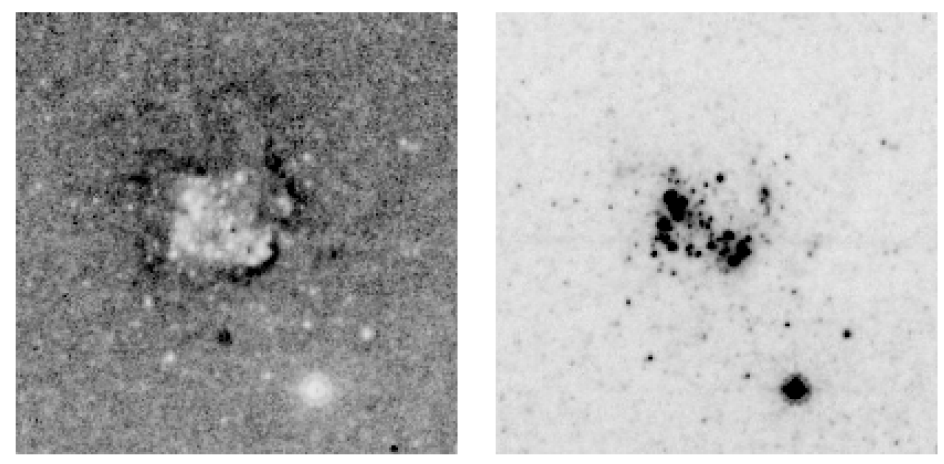

Fig. 10: On the left: nebula and star cluster cut off in Fig. 8. Image obtained by dividing images in the filters F658N and F814W. There is a visible gas shell around the cluster that has not dispersed due to the youth of the cluster. On the right: the same cluster in filter F814W.

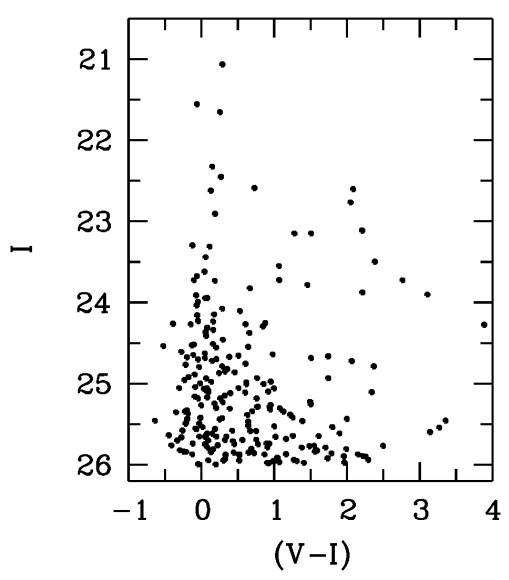

Fig. 11: CM diagram of young cluster from NGC 1672.

Stars that do not have exess in the $\mathrm{H} \alpha$ are marked in the diagram (Figure 7) with black squares. They are not of interest, although their luminocity may be even higher than that of stars with emission in $\mathrm{H} \alpha$.

Spectra for three stars with $\mathrm{H} \alpha$ emission (Figure 6) were obtained during the observations at the 6-m BTA (Solovyeva et al. 2019), which confirmed the presence of $\mathrm{H} \alpha$ line. These three stars change their luminosity and can be considered as LBV stars. No spectral observations were performed for the fourth star, but it can be assumed that this star also belongs to the class of bright massive stars.

The result of photometric search for massive stars in M94 have shown that such stars can be found using images in several filters.

The Seyfert galaxy NGC 1672 (Figure 8) is probably part of the Dorado group (Shobbrook 1966). Uncertainty arises from unreliable determination of distance. Using archival images of the Hubble Space Telescope (ID10354 and ID15654), we performed stellar photometry of this galaxy with the DAOPHOT II (Stetson 1987, 1994) and DOLPHOT 2.0 software packages. The selection of stars in DAOPHOT II was carried out in the same way as in DOLPHOT 2.0: $C H I<1.3$ and $|S H A R P|<0.3$. The resulting CM diagram of NGC 1672 stars is shown in Fig. 9a; Fig. 9b,c demonstrates the luminosity function, which 
The obtaned distance value ( $D=15.8 \pm 0.8 \mathrm{Mpc}$ ) confirms that NGC 1672 belongs to the Dorado group. The obtained distance allows us to measure the energy of the galactic nucleus, and to determine the luminosities of the brightest stars, which are visible on the obtained CM diagram (Figure 9).

Figure 8, a circle indicates a young star cluster surrounded by a gas shell (Figure 10) and containing stars up to $M_{V}=-10$. The CM diagram of this young cluster (Figure 11) does not differ from similar diagrams of other clusters. Therefore, we believe that most of the stars in this diagram are single stars and not cluster of stars. This young cluster is similar to the R136 cluster in BMO, where very massive stars are located.

Whether the bright stars of the cluster NGC 1672 are single or multiples can only be determined additional observations. Variability of the brightness of these stars would indicate that they are single stars. It should be noted that the stability of brightness does not prove that these stars are multiples or are star clusters.

\section{CONCLUSIONS}

The briefly described methods of searching of candidates for hypergiants are used by us for galaxies outside the Local Group. After compiling a list likely massive stars, we make spectral observation with a 6-m BTA telescope and obtain more detailed information about physical parameters of the stars. Not every galaxy contains bright hypergiants. To increase the width of the search for massive stars, we performed stellar photometry of the HST images of 320 galaxies in the northern sky. Possible hypergiants have been found in 53 galaxies. Planned spectral and photometric observations with ground-based telescopes will help us to find massive stars in these galaxies.

Acknowledgements The study was financially supported by the Russian Foundation for Basic Research and the National Science Foundation of Bulgaria as a part of the scientific project N 19-52-18007 and Grant KP-06-Russia-9/2019.

\section{References}

Bertelli, G., Bressan, A., Chiosi, C., Fagotto, F., \& Nasi, E. 1994, A\&AS, 106, 275 2, 3

Crowther, P. A., Schnurr, O., Hirschi, R., et al. 2010, MNRAS, 408, 7311

Dolphin, A. 2016, DOLPHOT: Stellar photometry 5

Eikenberry, S. S., Matthews, K., LaVine, J. L., et al. 2004, ApJ, 616, 5061

Garmany, C. D., \& Massey, P. 1981, PASP, 93, 5002

Gull, T. R. 2015, in Wolf-Rayet Stars, ed. W.-R. Hamann, A. Sander, \& H. Todt, 1492

Humphreys, R. M., Davidson, K., Hahn, D., Martin, J. C., \& Weis, K. 2017, ApJ, 844, 402

Pustilnik, S. A., Makarova, L. N., Perepelitsyna, Y. A., Moiseev, A. V., \& Makarov, D. I. 2017, MNRAS, 465,49852

Shobbrook, R. R. 1966, MNRAS, 131, 3657

Sholukhova, O. N., Fabrika, S. N., Valeev, A. F., \& Sarkisian, A. N. 2018, Astrophysical Bulletin, 73, 413 
Spera, M., \& Mapelli, M. 2017, MNRAS, 470, 47391

Stetson, P. B. 1987, PASP, 99, 1917

Stetson, P. B. 1994, PASP, 106, 2507

Tikhonov, N. A., Galazutdinova, O. A., \& Lebedev, V. S. 2014, Astronomy Letters, 40, 12

Yusof, N., Hirschi, R., Meynet, G., et al. 2013, MNRAS, 433, 11141 